discussion of mantle convection, which is an especial pity in view of the significant body of work on seismic mantle topography and its relation to the gravity field.

Another series of papers deals with the measurement of intercontinental baselines. Particularly useful is a paper on the Doppler tracking of satellites, for although the method is itself not very precise, it does provide a fairly uniform and continuous data set over nearly 20 years and we are beginning to see signatures of plate motions in these data. Missing here, unfortunately, is an account of the results from the laser tracking of satellites, but long-baseline interferometric methods are covered; although the data spans are very short, plate tectonic signals can be identified in these data as well. Baseline precision of a few centimetres, over lengths of several thousand kilometres, are being achieved, and it is only a matter of time and money before instantaneous plate motions can be monitored. It may take longer to understand them.

The movement of the Earth's rotation axis relative to the crust and to an inertial reference frame can be followed by a number of the new space methods with much greater accuracy than was previously possible. Several papers discuss these methods and results: one of the interesting results is the observation of some of the Earth's nutations which have led to the suggestion that the core-mantle boundary may depart from the hydrostatic equilibrium shape. The causes of irregularities in the rotation are considered in three papers, and much of the recent work is reviewed.

Overall, this book presents an up-todate summary of the status of space geodesy. The individual chapters offer balanced and reasonably detailed accounts of some of the main areas of the subject, and the reader comes away with an appreciation of the results and with a conviction that the long-held promises of the subject are about to be fulfilled. I read the book with a growing awareness of the progress made in the past two decades, and I look forward with excitement to a time when long series of measurements of the Earth's motion and deformation become available. When these are integrated with the geological evidence, we will then have a holistic description of the dynamics of the Earth.

Kurt Lambeck is Professor of Geophysics and Director of the Research School of Earth Sciences, Australian National University, PO Box 4, Canberra, ACT 2600, Australia.

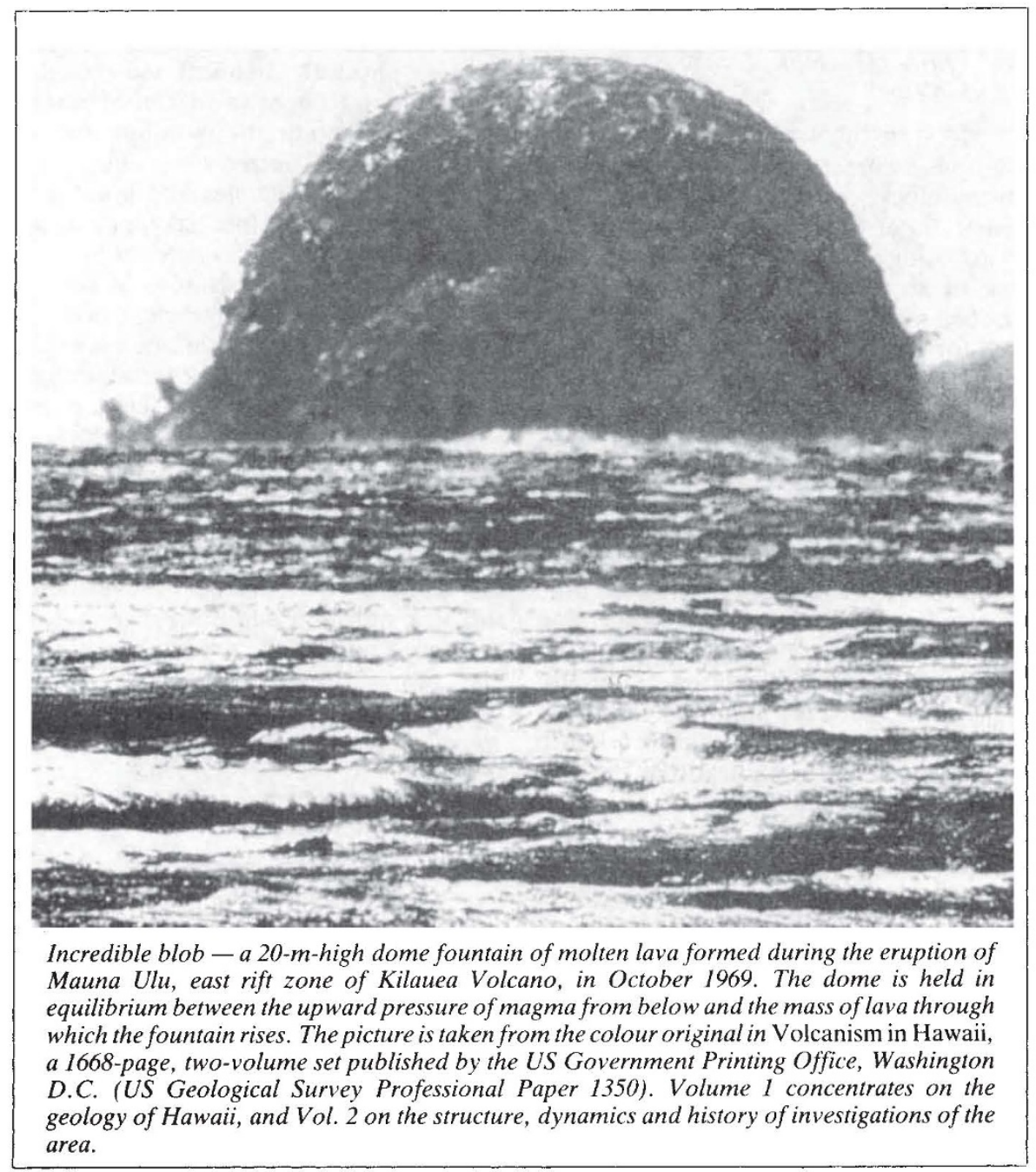

\section{Are desert reptiles different?}

\author{
Eric R. Pianka
}

Ecophysiology of Desert Reptiles. By S.D. Bradshaw. Academic:1987. Pp.324. Hbk $£ 29, \$ 58 ; p b k £ 17, \$ 34$.

THE modest energy requirements of reptiles enable them to capitalize on scant and unpredictable food resources, and these ectothermic animals - especially lizards - flourish in deserts. In his book, Bradshaw reviews aspects of the physiological ecology of certain desert reptiles, particularly two species of Australian agamid lizards, and gives comparative information, drawn from the literature, on some North American and Saharan lizards. The main emphasis is on water and electrolyte regulation, although thermoregulation, growth rates, reproductive tactics and life histories are also considered.

The author sets out to determine whether or not reptiles have evolved any special physiological features that enable them to cope with harsh desert conditions. The book begins with a brief review of the physical characteristics of deserts, followed by short discussions of the historical and biogeographical backgrounds of the herpetofaunae of Australian, North American and the Sahara deserts. The physiological and behavioural bases of lizard thermoregulation are briefly explored, with water and electrolyte balances being examined in some detail.

Lizards can tolerate substantial water losses and body chemistry varies profoundly between species; some, but not all, species possess salt excretory mechanisms. Similarly, reptilian reproductive tactics and life histories are diverse, but no uniquely desert patterns are identifiable. In many species, growth rates are variable between individuals. Case studies spanning three continents (Australia, northern Africa and North America) reveal some similarities, but also certain differences in how two species of agamid and one iguanid cope with life in the desert.

Bradshaw concludes that desert reptiles exhibit no obvious specific adaptations for living in arid places, but argues instead that the reptilian body plan is simply well suited for such environments. His book will be a useful reference for physiological ecologists.

Eric R. Pianka is the Denton A. Cooley Centennial Professor of Zoology at the University of Texas at Austin, Austin, Texas 78712, USA.

- Temperature Biology of Animals by A.R Cossins and K. Bowler deals with animal responses to temperature from the molecular to the whole organism and behavioural levels. The book is just published by Chapman \& Hall, price $£ 32.50, \$ 52.50$. 